\title{
A SUBARCSECOND RESOLUTION NEAR-INFRARED STUDY OF SEYFERT AND "NORMAL" GALAXIES. II. MORPHOLOGY
}

\author{
Johan H. KNAPen, ${ }^{1}$ IsAaC Shlosman, ${ }^{2}$ AND Reynier F. Peletier ${ }^{3}$ \\ Received 1999 May 21 ; accepted 1999 August 5
}

\begin{abstract}
We present a detailed study of the bar fraction in the CfA sample of Seyfert galaxies and in a carefully selected control sample of nonactive galaxies to investigate the relation between the presence of bars and of nuclear activity. To avoid the problems related to bar classification in the Third Reference Catalogue (RC3), e.g., subjectivity, low resolution, and contamination by dust, we have developed an objective bar classification method, which we conservatively apply to our new subarcsecond resolution near-infrared (NIR) imaging data set discussed in the first paper in this series. We are able to use stringent criteria based on radial profiles of ellipticity and major axis position angle to determine the presence of a bar and its axial ratio. Concentrating on noninteracting galaxies in our sample for which morphological information can be obtained, we find that Seyfert hosts are barred more often $(79 \% \pm 7.5 \%)$ than the nonactive galaxies in our control sample $(59 \% \pm 9 \%)$, a result which is at the $\sim 2.5 \sigma$ significance level. The fraction of nonaxisymmetric hosts becomes even larger when interacting galaxies are taken into account. We discuss the implications of this result for the fueling of central activity by large-scale bars. This paper improves on previous work by means of imaging at higher spatial resolution and by the use of a set of stringent criteria for bar presence and confirms that the use of NIR is superior to optical imaging for detection of bars in disk galaxies.
\end{abstract}

Subject headings: galaxies: evolution — galaxies: nuclei — galaxies: Seyfert — galaxies: spiral — galaxies: statistics — infrared: galaxies

\section{INTRODUCTION}

The study of fueling processes in active galactic nuclei (AGNs) is an issue important for our understanding of the structure and evolution both of central engines in AGNs and of their host galaxies. Although fuel is plentiful in the disk, it needs to overcome the centrifugal barrier to reach the innermost regions in disk and elliptical galaxies. Largescale nonaxisymmetries, such as galactic bars, are thought to be related to starburst activity within the central kiloparsec, which preferentially occurs in barred hosts (e.g., Heckman 1980; Balzano 1983; Devereux 1987; Kennicutt 1994). In a number of early optical surveys, the fueling of Seyfert activity in disk galaxies was linked to nonaxisymmetric distortions of galactic gravitational potentials by large-scale stellar bars and tidal interactions (Adams 1977; Heckman 1978; Simkin, Su, \& Schwarz 1980; Dahari 1984). This was supported by a more superficial argument that gravitational torques are able to remove the excess angular momentum from gas, which falls inward, giving rise to different types of activity at the center (see reviews by Shlosman 1992; Sellwood \& Wilkinson 1993; Phinney 1994). However, a criticism was leveled on observational results because the control samples were not matched to the Seyfert sample in properties like morphological distribution (Balick \& Heckman 1982; Fuentes-Williams \& Stocke 1988; Shlosman, Begelman, \& Frank 1990). More recent studies by Moles, Márquez, \& Pérez (1995) and Ho, Filippenko, \& Sargent (1997) conclude that the fraction of barred galaxies is equal among AGN hosts and the general

\footnotetext{
${ }^{1}$ Department of Physical Sciences, University of Hertfordshire, College Lane, Hatfield, Hertfordshire, AL10 9AB, England, UK; knapen@star.herts.ac.uk.

${ }_{2}$ Department of Physics and Astronomy, University of Kentucky, Lexington, KY 40506-0055; shlosman@pa.uky.edu.

${ }_{3}^{3}$ Department of Physics, University of Durham, South Road, Durham, DH1 3LE, England, UK; R.F.Peletier@dur.ac.uk.
}

population. However, these studies, like the ones in the past, continue to rely on (a) the morphological classification from optical catalogs (e.g., Third Reference Catalogue [RC3]: de Vaucouleurs et al. 1991) or (b) the use of control samples that are not matched to the "active" samples, e.g., the general population of spirals in the RC3.

A combination of dust obscuration, stellar populations, and inadequate spatial resolution can hide even a strong bar in the optical (Thronson et al. 1989, Block \& Wainscoat 1991; Spillar et al. 1992). High-quality near-infrared (NIR) imaging is much more reliable in determining the overall mass distribution in galaxies. Mulchaey \& Regan (1997) much improved the observational basis of this area of study, using NIR imaging of matched Seyfert and control samples of galaxies. Although their use of NIR imaging results in slightly higher bar fractions than, e.g., Ho et al. (1997), Mulchaey \& Regan do not find evidence for a significant excess of bars in Seyfert galaxies. Recent work by Regan \& Mulchaey (1999) involving Hubble Space Telescope (HST) color maps to search for the dust lane signatures of small-scale bars is potentially promising but employs a very small and statistically insignificant sample. It is also unclear whether nuclear bars have similar properties (e.g., dust lanes) to large-scale stellar bars and, hence, whether the criterion used is reliable.

The present paper aims to improve on past work through a combination of the following factors:

1. Use of NIR observations of a consistently high (subarcsecond) spatial resolution;

2. Use of a carefully selected control sample of nonactive galaxies;

3. Use of objective and stringent criteria for assigning bars and for determining bar axial ratio;

4. Publication of our complete set of images and profiles (Peletier et al. 1999, hereafter Paper I), to allow intercomparison with samples of other researchers. 
This paper is the second in a series analyzing the circumnuclear morphology and colors of a sample of Seyfert galaxies. Paper I describes the sample, the observations, data reduction, and photometric calibration and provides an analysis of the circumnuclear regions and host galaxy disks through color index maps. Paper III (Shlosman, Peletier, \& Knapen 1999) focuses on the distribution of bar axial ratios in Seyfert and nonactive galaxies. In the present paper (Paper II) we compare the morphology of the Seyfert sample with that of a control sample of nonactive galaxies, determining the fraction of nonaxisymmetric structures in AGN hosts.

In $\S 2$ we analyze the statistics of bars as determined from the RC3 and use that analysis in $\S 3$ to describe our Seyfert and control samples. Section 4 reviews the images and profiles of sample objects, as published in detail in Paper I, and describes our criteria for recognizing a bar in a given galaxy and for determining the bar's ellipticity. We present our results on bar fractions in $\S 5$ and compare them with other published work in $\S 6$. Finally, our results are discussed in terms of scenarios describing fueling of central activity by large-scale stellar bars in $\S 7$.

\section{BAR STATISTICS FROM THE RC3}

Before studying in detail the occurrence of bars in galaxies as determined from high-resolution NIR images, we consider statistics of bars as obtained from the RC3. Although NIR imaging has a better sensitivity to barred potentials, which we exploit in the rest of the paper, use of the RC3 has the advantage of sample size. Classifications for literally thousands of galaxies can be used to obtain indications on how bar fractions in galaxy populations vary as a function of various parameters.

Figure 1 shows how many galaxies are classified as "barred" (type X or B) in the RC3 by displaying the fraction of barred galaxies as a function of morphological type (Fig. 1, top), of ellipticity $\epsilon=1-b / a$ (equivalent to inclination, Fig. 1, center), and of recessional velocity (or redshift, Fig. 1, bottom). All parameters are directly obtained from the RC3.

Figure 1 confirms an overall bar fraction of $\sim 50 \%-60 \%$ (e.g., Sellwood \& Wilkinson 1993), but also shows that the bar fraction hardly varies with (1) morphological type for lenticular and spiral galaxies; (2) observed (i.e., not deprojected) ellipticity $\epsilon$, within the range of 0.1-0.4, and even 0.6 for the B-type galaxies; and (3) heliocentric velocity, or $z$. Due to the small numbers of galaxies at $v>10^{4} \mathrm{~km}$ $\mathrm{s}^{-1}$, the results of the distribution with recessional velocity are not conclusive, but do not show large systematic effects. As expected, the bar fraction is substantially lower for extremely early-type galaxies and for highly inclined galaxies $(\epsilon>0.6)$, where a bar will be much harder to recognize or classify.

Using the morphological classification of the complete set of galaxies in the RC3, we can determine the RC3 bar fractions in our Seyfert sample (see $\S 3$ for a description of the sample selection) and in a synthetic subsample of normal galaxies. The latter subsample was constructed from the RC3, with a distribution in terms of morphological type, ellipticity, and absolute magnitude similar to that of the Seyfert sample.

Since we have not encountered significant systematic effects in the RC3 classification within the parameter space under consideration here (see above), we will not at this
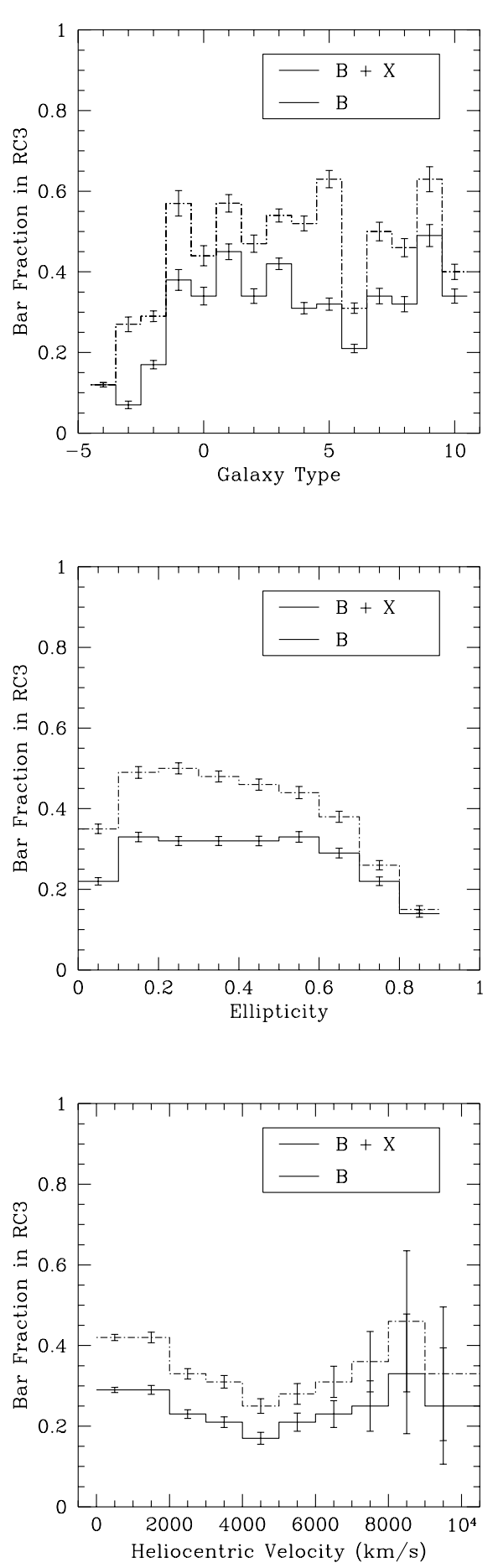

FIG. 1.-Bar fractions as determined from the RC3 morphological classification as a function of morphological type (top), galaxy ellipticity $(1-b / a)($ center $)$, and heliocentric velocity $($ bottom $)$.

stage correct the RC3 morphologies for systematic effects. We match the RC3 subsample to the Seyfert sample in the following way. For each CfA Seyfert galaxy of type $t_{i}$, ellipticity $\epsilon_{i}$, and absolute magnitude $M_{i}$, we select the galaxies in the RC3 with these parameters and determine the barred fraction $\left(f_{t_{i}, \epsilon_{i}, M_{i}}\right)$. The fraction of barred and mixed-type galaxies for the synthetic RC3 subsample thus constructed is given by $f_{\mathrm{B}, \mathrm{Sy}}=\Sigma_{i=1}^{n} f_{t_{i}, \epsilon_{i}, M_{i}} / n$, where $n$ is the number of Seyfert galaxies. 
Using the criteria outlined in more detail in $\S 4$, we excluded all galaxies for which one cannot perform a reliable bar classification. The CfA sample thus reduces from 48 to 29 Seyfert galaxies. Of these 29 , eight objects $(28 \% \pm 8 \%)$ have been classified in the RC 3 as type B and seven $(24 \% \pm 8 \%)$ as type $X$ (total B $+X 52 \% \pm 9 \%)$. For the corresponding synthetic RC3 subsample the fraction of B-type galaxies is $40 \% \pm 2 \%$ and of mixed type is $22 \% \pm 2 \%$ (total $\mathrm{B}+\mathrm{X} 62 \% \pm 2 \%$ ). Although there is no clear correspondence for individual galaxies between the RC3 morphological types and the bar axial ratios (Paper III; also R. J. Buta 1999, private communication), these numbers do indicate a tendency for B-type bars to be underabundant in the Seyfert sample, a finding which is upheld by studying other samples (Paper III).

We conclude that, based solely on the RC3 optical classification, there is a slight, and not necessarily significant, deficiency of barred galaxies among the Seyfert sample as compared to a synthetic control subsample with the same properties. This cannot be caused by any of the selection effects in morphological type, ellipticity, or absolute magnitude, as outlined above, because the two samples were explicitly matched using those criteria.

\section{OBSERVATIONS AND SAMPLE SELECTION}

We use the set of NIR imaging observations of the (CfA) sample of Huchra \& Burg (1992) for the Seyfert galaxies, as described in Paper I. We summarize only the basic details here. We observed the complete CfA sample in the NIR $J$, $H$, and $K$ bands, apart from $3 C 273$, which was not included because of its high redshift $(0.16$, vs. 0.07 for the second highest). Observations were made under good seeing conditions (spatial resolution of less than $1^{\prime \prime}$, best seeing 0.55 , with median over all images 0.7 ). The galaxy exposures were interleaved with sky exposures, and standard stars were observed for the photometric calibration.

The data of our sample of 48 Seyfert galaxies are compared with $K$-band images of the same quality of a control sample of 34 galaxies, selected from the RC3 to mimic the Seyfert sample in terms of morphological type and ellipticity. Full details of the observations and data reduction can be found in Paper I, where we also publish all of the images and derived surface brightness, color, ellipticity, and position angle profiles for both Seyfert and non-Seyfert samples. We made sure none of our control galaxies is active by cross-checking with different sources of AGN classification. In Figure 2 we provide the distribution of both samples as a function of morphological type, galaxy ellipticity, and redshift. One important difference between the two samples, obvious in these figures, is that a significant fraction of the Seyfert galaxies does not have a well-defined type in the RC3. For such galaxies, as well as for those at high inclination, or large $z$, determination of the presence and axial ratios of bars is difficult or impossible, and we thus proceeded by removing those galaxies from our sample.

We exclude from our analysis those galaxies that are

1. too small to study their morphology, as determined from their radius (in arcseconds) at a surface brightness level of $19 \mathrm{H}$ mag $\operatorname{arcsec}^{-1}$ : if $\log r_{H, 19}<0.8$; such galaxies have been marked with code 1 in Tables 1 and 2;

2. strongly interacting, as evident either by a severely
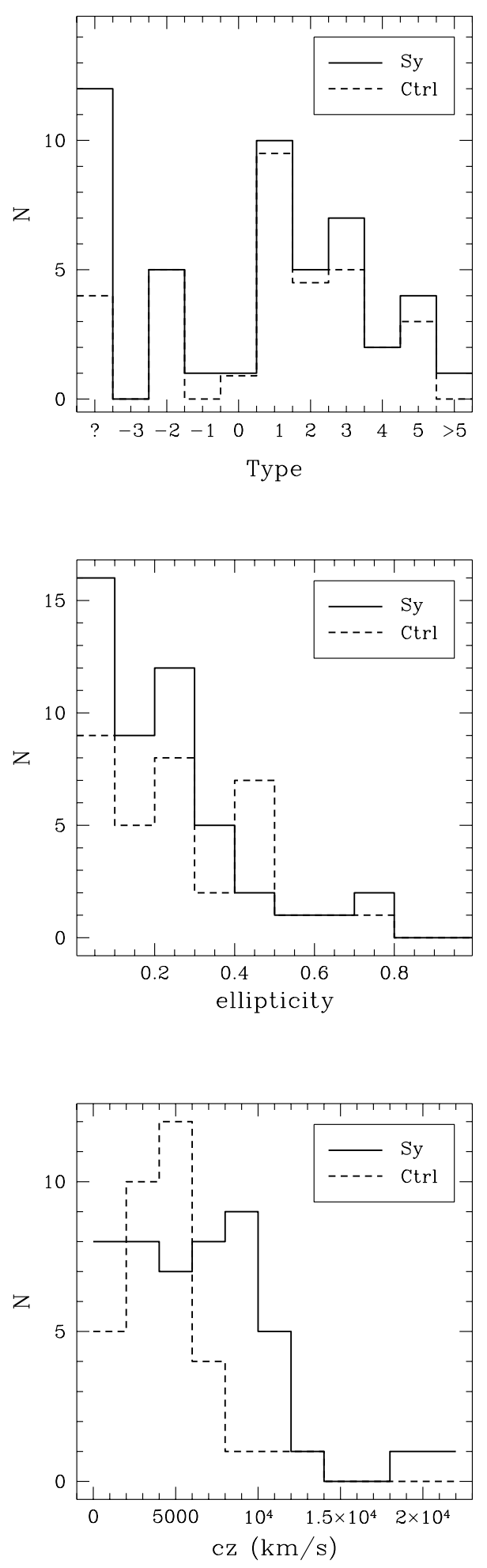

Fig. 2.-Distribution of Seyfert and control sample before removing the galaxies for which no reliable bar classification could be made.

distorted morphology or by the presence of a companion within $1^{\prime}$ (code 2);

3. highly inclined, with $\epsilon>0.5$ (see Fig. 1, center) (code $3)$.

We chose the limits in such a way as to disqualify those galaxies for which the information on the isophote shapes as derived from the imaging would not lead to reliable state- 
TABLE 1

Parameters for the Seyfert SAmple GalaXies

\begin{tabular}{|c|c|c|c|c|c|c|c|}
\hline \multirow[b]{2}{*}{$\begin{array}{l}\text { GALAXY } \\
\text { (1) }\end{array}$} & \multirow[b]{2}{*}{$\begin{array}{l}v_{\text {hel }} \\
(2)\end{array}$} & \multirow[b]{2}{*}{$\begin{array}{c}\text { SEYFERT } \\
\text { (3) }\end{array}$} & \multirow[b]{2}{*}{$\begin{array}{l}\text { TYPE } \\
\text { (4) }\end{array}$} & \multirow[b]{2}{*}{$\begin{array}{l}\text { Class } \\
\text { (5) }\end{array}$} & \multicolumn{2}{|c|}{ BAR "STRENGTH" } & \multirow[b]{2}{*}{$\begin{array}{c}\text { CoDE } \\
(8)\end{array}$} \\
\hline & & & & & $\begin{array}{l}\text { Projected } \\
\text { (6) }\end{array}$ & $\begin{array}{c}\text { Deprojected } \\
\text { (7) }\end{array}$ & \\
\hline Mrk $334 \ldots \ldots \ldots$ & 6582 & 1.8 & $\mathrm{~N}$ & $. P \ldots . .$. & & & 2 \\
\hline Mrk $335 \ldots \ldots \ldots$ & 7688 & 1 & $\mathrm{~N}$ &.$P \ldots \ldots$ & & & 1 \\
\hline UGC $524 \ldots \ldots \ldots$ & 10763 & 1 & 3.0 & PSBS3.. & 6 & 6 & \\
\hline I $\mathrm{Zw}_{\mathrm{w}} 1 \ldots \ldots \ldots \ldots$ & 18330 & 1 & $\mathrm{~N}$ &.$S ? \ldots$ & & & 1 \\
\hline Mrk $993 \ldots \ldots \ldots$ & 4658 & 2 & 1.0 & .S..1.. & & & 3 \\
\hline Mrk $573 \ldots \ldots \ldots$ & 5174 & 2 & -1.0 & RLXT +*. & ND & ND & 4 \\
\hline UGC $1395 \ldots \ldots \ldots$ & 5208 & 1.9 & 3.0 & .SAT3.. & 6 & 5 & \\
\hline Mrk $590 \ldots \ldots \ldots$ & 7910 & 1.2 & 1.0 & .SAS1*. & ND & ND & 4 \\
\hline NGC $1068 \ldots . . .$. & 1136 & 2 & 3.0 & RSAT3.. & 5 & 4 & \\
\hline NGC $1144 \ldots . .$. & 8648 & 2 & $\mathrm{~N}$ & .RING.B & & & 2 \\
\hline Mrk $1243 \ldots \ldots \ldots$ & 10602 & 1 & 1.0 & .S..1.. & & & 1 \\
\hline NGC $3227 \ldots \ldots$. & 1157 & 1.5 & 1.0 & .SXS1P. & & & 2,3 \\
\hline NGC $3362 \ldots \ldots \ldots$ & 8318 & 2 & 5.0 &.$S X .5$. & 4 & 2 & \\
\hline UGC $6100 \ldots . .$. & 8778 & 2 & 1.0 & $. S . .1 ?$. & & & \\
\hline NGC $3516 \ldots . . .$. & 2649 & 1.5 & -2.0 & RLBS0*. & 3 & 4 & \\
\hline Mrk $744 \ldots \ldots \ldots$ & 2674 & 1.8 & 1.0 & .SXT1P. & & & 2,3 \\
\hline NGC $3982 \ldots \ldots \ldots$ & 1109 & 2 & 3.0 & .SXR3*. & 4 & 3 & \\
\hline NGC $4051 \ldots . .$. & 725 & 1 & 4.0 & .SXT4.. & 7 & 6 & \\
\hline NGC $4151 \ldots \ldots$. & 995 & 1.5 & 2.0 & PSXT2*. & 5 & 3 & \\
\hline NGC $4235 \ldots \ldots \ldots$ & 2410 & 1 & 1.0 & .SAS1./ & & & 3 \\
\hline Mrk $766 \ldots \ldots \ldots$ & 3876 & 1.5 & 1.0 & PSBS1*. & 6 & 6 & \\
\hline Mrk $205 \ldots \ldots \ldots$ & 21239 & 1 & $\mathrm{~N}$ & $. P \ldots .$. & & & 1 \\
\hline NGC $4388 \ldots . .$. & 2524 & 2 & 3.0 & .SAS3*/ & & & 3 \\
\hline NGC $4395 \ldots \ldots \ldots$ & 319 & 1.8 & 9.0 & .SAS9*. & 7 & 7 & \\
\hline Mrk $231 \ldots \ldots \ldots$ & 12642 & 1 & 5.0 &. SAT5\$P & & & 2 \\
\hline NGC $5033 \ldots . .$. & 875 & 1.9 & 5.0 & .SAS5.. & & & 3 \\
\hline Mrk $789 \ldots \ldots \ldots$ & 9476 & 1 & $\mathrm{~N}$ & $. P \ldots . .$. & & & 1,2 \\
\hline UGC $8621 \ldots \ldots$. & 6023 & 1.8 & $\mathrm{~N}$ &.$S ? \ldots$. & & & \\
\hline NGC $5252 \ldots . .$. & 6926 & 1.9 & -2.0 & $. \mathrm{L} \ldots . .$. & 6 & 4 & \\
\hline Mrk $266 \ldots \ldots \ldots$ & 8353 & 2 & $\mathrm{~N}$ &.$P \ldots \ldots$ & & & 2 \\
\hline Mrk $270 \ldots \ldots \ldots$ & 2700 & 2 & -2.0 &.$L \ldots ?$ ? & ND & ND & 4 \\
\hline NGC $5273 \ldots \ldots$. & 1089 & 1.9 & -2.0 & .LAS0.. & & & \\
\hline Mrk $461 \ldots . . .$. & 4856 & 2 & $\mathrm{~N}$ &. S...... & & & \\
\hline NGC $5347 \ldots \ldots$. & 2335 & 2 & 2.0 & PSBT2.. & 7 & 6 & \\
\hline Mrk $279 \ldots \ldots \ldots$ & 8814 & 1 & -2.0 & $. \mathrm{L} \ldots . .$. & 4 & 2 & \\
\hline NGC $5548 \ldots \ldots \ldots$ & 5149 & 1.5 & 0.0 & PSAS0.. & & & \\
\hline NGC $5674 \ldots . .$. & 7474 & 1.9 & 5.0 &. $\mathrm{SX} .5$. & 6 & 6 & \\
\hline Mrk $817 \ldots \ldots \ldots$ & 9430 & 1.5 & $\mathrm{~N}$ &.$S ? \ldots$ & 5 & 5 & \\
\hline Mrk $686 \ldots \ldots \ldots$ & 4225 & 2 & 3.0 & .SB.3.. & 5 & 4 & \\
\hline Mrk $841 \ldots \ldots \ldots$ & 10852 & 1 & $\mathrm{~N}$ & ......... & & & 1 \\
\hline NGC $5929 \ldots . .$. & 2492 & 2 & 2.0 & $. S . .2 * \mathrm{P}$ & & & 2 \\
\hline NGC $5940 \ldots . . .$. & 10115 & 1 & 2.0 & .SB.2.. & 5 & 5 & \\
\hline NGC $6104 \ldots \ldots$. & 8382 & 1.5 & $\mathrm{~N}$ &.$S ? \ldots$ & & & 2 \\
\hline UGC $12138 \ldots . .$. & 7375 & 1.8 & 1.0 & .SB.1.. & 6 & 5 & \\
\hline NGC $7469 \ldots . .$. & 4892 & 1 & 1.0 & PSXT1.. & 4 & 2 & \\
\hline Mrk $530 \ldots \ldots \ldots$ & 8851 & 1.5 & 3.0 &. $\mathrm{SAT} 3 * \mathrm{P}$ & & & \\
\hline Mrk $533 \ldots \ldots \ldots$ & 8713 & 2 & 4.0 & .SAR4P. & & & 2 \\
\hline NGC $7682 \ldots \ldots \ldots$ & 5107 & 2 & 2.0 & .SBR2.. & 6 & 6 & \\
\hline
\end{tabular}

Note.-Col. (1): Galaxy names. Col. (2): Heliocentric radial velocity. Col. (3): Seyfert classification from NASA's Extragalactic Database (NED). Col. (4): Numerical type from RC3. Col. (5): Morphological type from RC3. Col. (6): Bar strength (projected) as determined from the ellipticity profiles (see text). Code ND in this column means galaxy is barred but no bar strength could be determined. Col. (7): Deprojected bar strength. Col. (8): Codes indicate whether galaxies belong to any of the following groups: 1: Too small to study morphology $\left(\log r_{H, 19}<0.8\right.$ with $r$ in arcminutes); 2 : Strongly interacting - severely distorted or companion within $1^{\prime} ; 3$ : Edge-on $(\epsilon>0.5)$; 4: P.A. twist $>75^{\circ}$. Codes 1,2 , or 3 disqualify from further statistical analysis; code 4 indicates presence of bar. 
TABLE 2

Parameters for the Control Sample Galaxies

\begin{tabular}{|c|c|c|c|c|c|c|}
\hline \multirow[b]{2}{*}{$\begin{array}{l}\text { GALAXY } \\
\text { (1) }\end{array}$} & \multirow[b]{2}{*}{$\begin{array}{l}v_{\text {hel }} \\
(2)\end{array}$} & \multirow[b]{2}{*}{$\begin{array}{c}\text { TYPE } \\
(3)\end{array}$} & \multirow[b]{2}{*}{$\begin{array}{l}\text { CLASS } \\
\text { (4) }\end{array}$} & \multicolumn{2}{|c|}{ BAR “STRENGTH" } & \multirow[b]{2}{*}{$\begin{array}{l}\text { CoDE } \\
\text { (7) }\end{array}$} \\
\hline & & & & $\begin{array}{l}\text { Projected } \\
\text { (5) }\end{array}$ & $\begin{array}{l}\text { Deprojected } \\
\text { (6) }\end{array}$ & \\
\hline NGC $1093 . . . .$. & 6282 & 2.0 &.$S X .2 ?$ & 7 & 6 & \\
\hline UGC $3247 \ldots \ldots$. & 3371 & $\mathrm{~N}$ &.$S ? \ldots$. & 5 & 5 & \\
\hline UGC $3407 \ldots \ldots$. & 3604 & 1.0 & .S..1.. & 4 & 4 & \\
\hline UGC $3463 \ldots \ldots$ & 2693 & 4.0 &. SXS4.. & & & \\
\hline UGC $3536 \ldots \ldots$ & 4689 & -3.0 & $. \mathrm{L} \ldots .$. & 6 & 2 & \\
\hline UGC $3576 \ldots \ldots$ & 5966 & 3.0 & .SBS3.. & 6 & 3 & \\
\hline UGC $3592 \ldots \ldots$ & 13160 & 1.0 & RSBS1.. & 7 & 8 & \\
\hline UGC $3789 \ldots \ldots$ & 3325 & 2.0 & RSAR2.. & 6 & 6 & \\
\hline UGC $3850 \ldots \ldots$ & 4709 & 1.0 & PSXS1.. & 5 & 5 & \\
\hline NGC $2347 \ldots . .$. & 4421 & 3.0 & PSAR3*. & & & \\
\hline NGC $2365 . . . .$. & 2278 & 1.0 & .SX.1.. & & & \\
\hline NGC $2431 \ldots . .$. & 5679 & 1.0 & PSBS1*. & ND & ND & 4 \\
\hline NGC $2460 \ldots . .$. & 1442 & 1.0 & .SAS1.. & & & \\
\hline NGC $2487 \ldots \ldots$. & 4841 & 3.0 & .SB.3.. & 6 & 5 & \\
\hline NGC $2599 . . . .$. & 4741 & 1.0 & .SA.1.. & & & \\
\hline NGC $2855 \ldots . .$. & 1910 & 0.0 & RSAT0.. & 3 & 2 & \\
\hline NGC $3066 \ldots . .$. & 2049 & 4.0 & PSXS4P. & 5 & 5 & \\
\hline NGC $3188 \ldots \ldots$. & 7769 & 2.0 & RSBR2.. & 6 & 6 & \\
\hline NGC $3455 \ldots \ldots$. & 1102 & 3.0 & PSXT3.. & & & \\
\hline NGC $4146 \ldots . .$. & 6520 & 1.5 & RSXS2.. & 5 & 5 & \\
\hline NGC $4369 . . . .$. & 1045 & 1.0 & RSAT1.. & 7 & 7 & \\
\hline NGC $4956 \ldots . .$. & 4750 & -2.0 & $. L \ldots . .$. & & & \\
\hline NGC $4966 \ldots . .$. & 7036 & $\mathrm{~N}$ & $. S \ldots . .$. & & & \\
\hline NGC $5434 \ldots . .$. & 5638 & 5.0 & .SA.5.. & & & 2,4 \\
\hline NGC $5534 \ldots . .$. & 2633 & 1.7 & PSXS2P* & & & 2 \\
\hline NGC $5832 \ldots \ldots$. & 447 & 3.0 & .SBT3\$. & & & \\
\hline NGC $5869 \ldots . .$. & 2087 & -2.0 & .L... $0 *$. & ND & ND & 4 \\
\hline UGC $9965 \ldots \ldots$ & 4528 & 5.0 & .SAT5.. & 5 & 4 & \\
\hline NGC $5992 \ldots . .$. & 9518 & $\mathrm{~N}$ & $. \mathrm{S} . . . .$. & & & 2 \\
\hline NGC $6085 \ldots . .$. & 10195 & 1.0 & .S..1.. & & & \\
\hline NGC $6278 \ldots \ldots$ & 2790 & -2.0 & $. \mathrm{L} \ldots . . .$. & & & \\
\hline NGC $6504 \ldots . .$. & 4788 & $\mathrm{~N}$ & $. \mathrm{S} \ldots . . .$. & & & 3 \\
\hline NGC $6635 \ldots . .$. & 5038 & -2.0 &.$L \ldots P^{*}$ & & & \\
\hline NGC $6922 \ldots . .$. & 5665 & 5.3 & .SAT5P* & & & 2 \\
\hline
\end{tabular}

NotE. - Col. (1): Galaxy names. Col. (2): Heliocentric radial velocity. Col. (3): Numerical type from RC3. Col. (4): Morphological type from RC3. Col. (5): Bar strength (projected) as determined from the ellipticity profiles (see text). Code ND in this column means galaxy is barred but no bar strength could be determined. Col. (6): Deprojected bar strength. Col. (7): Codes indicate whether galaxies belong to any of the following groups: 1: Too small to study morphology $\left(\log r_{H, 19}<0.8\right.$ with $r$ in arcminutes); 2 : Strongly interacting - severely distorted or companion within $1^{\prime} ; 3$ : Edge-on $(\epsilon>0.5)$; 4: P.A. twist $>75^{\circ}$. Codes 1,2 , or 3 disqualify from further statistical analysis; code 4 indicates presence of bar.

ments on the presence of a bar. The final statistical results do not critically depend on fine-tuning these numerical limits.

In Figure 3 we show the distributions for the reduced sample, which we use in the statistical analysis (§5). Although there are still some minor differences between the reduced Seyfert and non-Seyfert samples in terms of morphological type, ellipticity, and redshift, such differences are small. In any case, the remaining differences are unlikely to affect our statistical results on bar fractions because, as we have shown in Figure 1, there are no systematic trends of bar fraction as a function of these parameters over the range spanned in type, ellipticity, and $z$.

\section{BAR CRITERIA AND DETERMINATION OF AXIAL RATIOS}

Our main criterion for the presence of a bar is a significant rise in the radial ellipticity profile of a galaxy (Paper I) followed by a significant fall over a range in radius where the position angle of the major axis is roughly constant. The amplitude of the ellipticity variation must be at least 0.1 .

We also classify galaxies as barred if their radial majoraxis position angle profile (Paper I) shows a change of more than $75^{\circ}$, accompanied by ellipticity values above roughly 0.1 . Such NIR isophote twists are characteristic of the inner disk resonance region where nuclear rings are found (Shaw et al. 1993; Knapen et al. 1995; Heller \& Shlosman 1996). Furthermore, large isophote twists $\left(>50^{\circ}\right)$ are very uncommon in elliptical galaxies, and hence, it is very unlikely that they are related to the possible triaxiality of the central bulges in disk galaxies (Peletier et al. 1990).

We primarily used our NIR imaging, but since most galaxies are larger than our field of view, we extended the radial range of the ellipticity and position angle profiles by using images from the digitized sky survey (see Paper I). This allows the detection of large-scale bars, for which our 

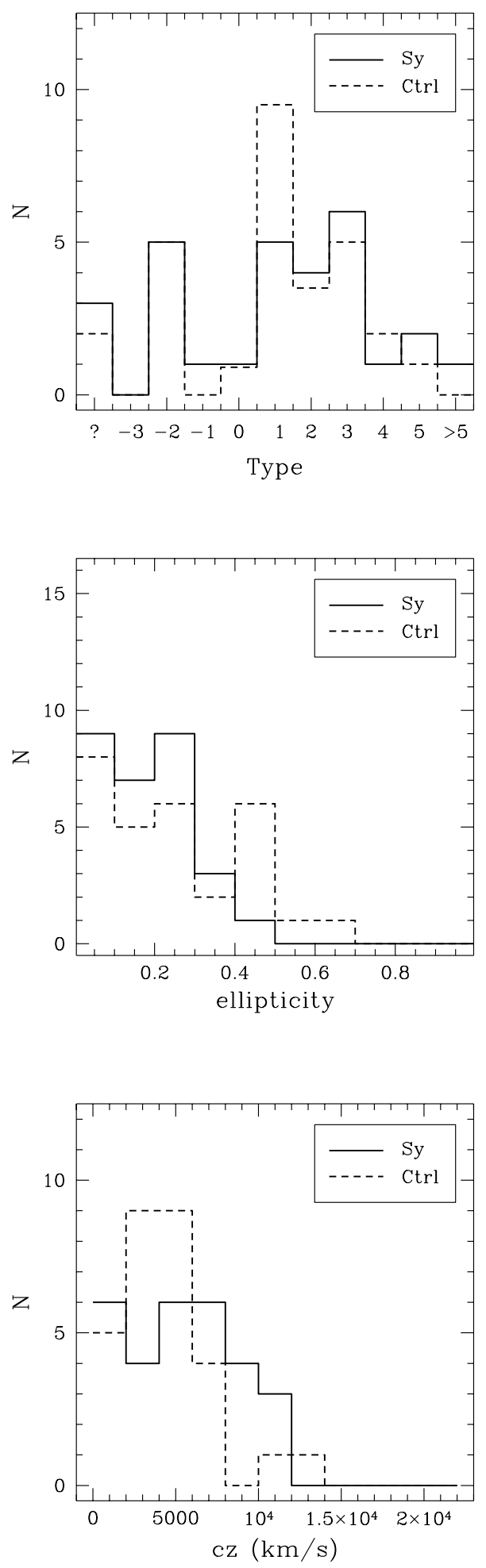

FIG. 3.-Distribution of Seyfert and control sample after removing the galaxies for which no reliable bar classification could be made.

NIR data alone would not be sufficient to confirm the decrease in ellipticity beyond the range of the bar.

In a number of cases, we suspect the presence of a bar in a galaxy, but the results obtained from our data do not satisfy our stringent bar criteria. We have thus counted these galaxies as nonbarred in our statistics, but data of higher quality may well lead to their reclassification as barred gal- axies. This group includes as most prominent members Mrk 461 and UGC 8621 in the Seyfert sample and NGC 3455 and NGC 4966 in the control sample.

We determined axial ratios of the bars from the ellipticity profiles using the criterion put forward by Martin (1995), where $\epsilon_{b}=10(1-b / a)$, thus "no bar" means $\epsilon_{b}=0$ and a very "strong" bar $\epsilon_{b}=8$. The term $\epsilon_{b}$ is read off from the profile as the maximum $\epsilon$ at the bar position and is subsequently corrected for the inclination of the galaxy. The bar ellipticity is used here in a narrow sense, namely, to define the axial ratios. Realistic bars, of course, can differ substantially from ideal elliptical shapes.

Tables 1 and 2 contain information on the presence of bars in our sample galaxies and on the maximum axial ratios of those bars. An entry in the "Bar 'Strength" column indicates the presence of a bar; a blank in that column accompanied by a blank in the last column ("Code") means the galaxy is nonbarred in our imaging study.

\section{RESULTS}

\subsection{CfA Seyfert Sample}

After disqualifying galaxies due to their small angular size, interaction, or high inclination, our Seyfert sample is reduced from 48 galaxies to 29 . Of these 29 galaxies, 20 have a bar as determined from the ellipticity profile, and for all of these we can determine the bar axial ratio. Another three galaxies are barred as determined from their position angle twist, but for these the bar axial ratios cannot be determined. We conclude that 23 out of 29 galaxies are barred, or $79 \% \pm 7.5 \%$ ( $1 \sigma$ Poisson error $)$.

Mrk 270 has a small bar at $r<2^{\prime}$, but since this lies at the edge of our resolution limit (about 3 times the seeing value) we have taken the convincing position angle twist at larger radii as prime evidence for the presence of a bar.

\subsection{Control Sample}

Following the same approach as for the Seyfert galaxies, we first exclude five of our 34 galaxies due to interactions or high inclination. Of the remaining 29,17 are found to be barred, or $59 \% \pm 9 \%$. We could determine the bar axial ratios for all but two of these galaxies.

\section{COMPARISON WITH PUBLISHED RESULTS}

As outlined in the introduction, the methodology used in the present paper is very different from that used in most related published studies, with the exception of Mulchaey \& Regan (1997). All other authors used morphological classifications as taken at face value from one of the main galaxy catalogs. Furthermore, in many studies, an active galaxy population is compared with a control population that is not matched in detail.

Ho et al. (1997) use the results from their spectroscopic survey of a large magnitude-limited sample of galaxies to study the relations between bar fraction and nuclear activity. Information on activity in their sample galaxies is obtained from this spectral survey, whereas morphological information on the host galaxy is taken from the RC3. Ho et al. consider an AGN subsample, which includes LINER/H II transition objects, LINERs, and Seyfert galaxies, and compare its bar fraction with that of the complete sample. They find that the bar fraction in the AGN sample 
is slightly lower, but not significantly so $(57 \% \pm 4 \%$ for the AGN sample vs. $61 \% \pm 4 \%$ ).

Mulchaey \& Regan (1997) invoked a similar (but not identical in details) approach to the one described by us here, namely, analyzing NIR images of matching samples of Seyfert and nonactive galaxies. To determine the presence and axial ratios of the bars, they use in the first instance radial ellipticity and position angle profiles (as published by Mulchaey, Regan, \& Kundu 1997) but, unlike in the present study, add a few cases where the bar is "visible" on the image but not in the profiles. Mulchaey \& Regan (1997) conclude that the galaxies in both their Seyfert and control samples are barred in just over $70 \%$ of all cases. Based on the size of their samples, the errors in these numbers should be around $8 \%$. Considering these uncertainties, their result is not in contradiction with results of this study.

The main improvement in our work is the use of images at consistently higher resolution than Mulchaey \& Regan's (median seeing of our data 0.7 , vs. 1.0 , with variations between 0.8 and 1".8, for Mulchaey \& Regan 1997). Some other differences between the study of Mulchaey \& Regan (1997) and ours that might influence the resulting bar fractions can be identified. First, Mulchaey \& Regan use a subset of the incomplete sample of Maiolino, Ruiz, \& Rieke (1995), essentially all known Seyfert galaxies in the Revised Shapley-Ames catalog (RSA) (Sandage \& Tammann 1981). The CfA sample, on the other hand, is considered to be an optically complete set of Seyfert galaxies in a region of the sky. Second, Mulchaey \& Regan match their active and control samples using, among other parameters, the absolute blue magnitude, whereas we chose not to. The advantage of Mulchaey \& Regan's approach is that the two samples are better matched in distance; but, the uncertainty of the fraction of total blue light attributed to the galaxy by the AGN and the potentially related fact that Seyfert host galaxies tend to be more luminous than average spirals (Mulchaey \& Regan 1997), in our opinion, offset this advantage. Third, whereas Mulchaey \& Regan, in a few cases, update their bar classification on the basis of a visual inspection, even if the ellipticity and position angle profiles do not convincingly show the presence of a bar, we have stubbornly maintained our stringent criteria for bar presence based upon the behavior of the radial profiles. This may result in a lower bar fraction than that found by Mulchaey \& Regan, as is in fact the case in the control sample. Our higher bar fraction in the Seyfert sample, then, is further proof of the power of a modest increase in spatial resolution in the NIR imaging.

We adjust the classification given by McLeod \& Rieke (1995) in two cases: NGC 3982, classified as .SXR3*. [or $\mathrm{SAB}(\mathrm{r}) \mathrm{b}]$ in the RC3 (but not barred according to McLeod \& Rieke), does in fact have a bar inside a region of welldefined spiral arms; and Mrk 817 has a nice but angularly small bar.

We have cross-checked our results for the presence of bars in the Seyfert host galaxies with those presented by Malkan, Gorjian, \& Tam (1998). The following galaxies in our CfA sample were classified as barred by Malkan et al.: Mrk 993 (disqualified by us due to its high inclination), Mrk 766, NGC 5674, Mrk 817, NGC 5940, NGC 6104 (disqualified by us for interaction), UGC 12138, Mrk 533 (disqualified by us for interaction), and NGC 7682. Apart from the three galaxies we disqualified, all these galaxies are confirmed to be barred in our study.
An extensive comparison of bar axial ratios as determined by us and taken from the literature, statistically and in individual cases, is presented in Paper III.

\section{CONCLUDING REMARKS: LARGE-SCALE STELLAR BARS AND FUELING OF AGNs}

Based on our new subarcsecond resolution NIR imaging survey of the CfA sample of Seyfert galaxies and of a control sample of normal galaxies, and excluding objects for which reliable morphological information cannot be obtained, we find that Seyfert hosts are barred more often than normal galaxies $(79 \% \pm 7.5 \%$ barred for the Seyfert galaxies, versus $59 \% \pm 9 \%$ for the control sample). This result stands at the $2.5 \sigma$ level.

Taking the bar classification from the $\mathrm{RC} 3$, we find that Seyfert galaxies are barred just as often as normal galaxies, in general agreement with previous work (e.g., Simkin et al. 1980; Moles et al. 1995; Ho et al. 1997), also based upon the RC3. The classification in the RC3 was done by eye on the basis of photographic plates taken in the optical and at relatively poor angular resolution, and although performed by experts, the classification remains difficult to reproduce for individual galaxies. This is illustrated, for example, by Sellwood \& Wilkinson (1993), who compare bar fractions based on several catalogs, and by the present work (see Tables 1 and 2).

In contrast, in our new study we observe the morphology of active and nonactive galaxies in the NIR, unhampered by dust, and at a much higher resolution than previous work, including the other NIR studies in this field by McLeod \& Rieke (1995) and Mulchaey \& Regan (1997). Our result, at a significance level of $\sim 2.5 \sigma$, suggests that there is an underlying morphological difference between Seyfert and nonSeyfert galaxies. Since the large error margins are a direct result of the sample size, it is now imperative to check the validity of our observational conclusion with larger samples.

A few comments follow from the above statistics. First, these results emphasize the prevalence of barred morphologies in disk galaxies in general, and in active galaxies in particular. It is known from extensive theoretical studies that nonaxisymmetric potentials induce radial gas flows and elevate dramatically the rate of star formation within the central kiloparsec of barred galaxies. A straightforward extrapolation toward much smaller spatial scales characterizing the nonstellar AGN-type activity is not warranted, however. The absence of AGNs in many barred galaxies can be interpreted in terms of additional factors besides the large-scale stellar bars, which are required to trigger the nuclear nonstellar activity, a point emphasized already by Shlosman, Frank, \& Begelman (1989). These additional factors should include the availability of fuel, efficiency of star formation, and global self-gravitating effects in the circumnuclear gas, underlining the increasingly important role the gas plays in galactic dynamics at progressively smaller radii (Shlosman 1996).

To summarize, our new NIR data set improves on earlier published surveys of the CfA Seyfert sample in the following aspects: (1) complete coverage of the sample, (2) observations in the NIR $J, H$, and $K$ bands of all objects, (3) improved spatial resolution: $<1^{\prime \prime}$ for all our images, best seeing 0.55 , with median over all images 0.7 , and (4) observations of a matched control sample in the $K$ band. In addition, there is no reliance on optically selected galaxy 
catalogs, and the morphology of both the CfA and the control sample galaxies was critically revised. At the resolution level of the $R C 3$ optical catalog, there is an agreement between our results and those of past and recent surveys (e.g., Simkin et al. 1980; Moles et al. 1995; Ho et al. 1997). It is the high NIR resolution used here that led us to our main conclusion.
We thank Ron Buta, Françoise Combes, and Bill Keel for helpful communications. We made use of the NASA/IPAC Extragalactic Database (NED) which is operated by Jet Propulsion Laboratory, California Institute of Technology, under contract with NASA. I. S. is supported in part by NASA grants NAGW-3841, WKU-522762-98-06, G008123.01-97A, and HST AR-07982.01-96A.
Adams, T. F. 1977, ApJS, 33, 19

Balick, B., \& Heckman, T. M. 1982, ARA\&A, 20, 431

Balzano, V. A. 1983, ApJ, 268, 602

Block, D. \& Wainscoat, R. J. 1991, Nature, 353, 48

Dahari, O. 1984, Ph.D. thesis, Univ. California, Santa Cruz

de Vaucouleurs, G., de Vaucouleurs, A., Corwin, H. G., Buta, R. J., Paturel,

G., \& Fouqué, P. 1991, Third Reference Catalogue of Bright Galaxies (RC3) (New York: Springer)

Devereux, N. A. 1987, ApJ, 323, 91

Fuentes-Williams, T., \& Stocke, J. T. 1988, AJ, 96, 1235

Heckman, T. 1978, PASP, 90, 241 1980, A\&A, 88, 365

Heller, C. H., \& Shlosman, I. 1996, ApJ, 471, 143

Ho, L. C., Filippenko, A. V., \& Sargent, W. L. W. 1997, ApJ, 487, 591

Huchra, J. P., \& Burg, R. 1992, ApJ, 393, 90

Kennicutt, R. C. 1994, in Mass-Transfer Induced Activity in Galaxies, ed. I. Shlosman (New York: Cambridge Univ. Press), 131

Knapen, J. H., Beckman, J. E., Heller, C. H., Shlosman, I., \& de Jong, R. S. 1995, ApJ, 454, 623

Maiolino, R., Ruiz, M., \& Rieke, G. H. 1995, ApJ, 446, 561

Malkan, M. A., Gorjian, V., \& Tam, R. 1998, ApJS, 117, 25

Martin, P. 1995, AJ, 109, 2428

McLeod, K. K., \& Rieke, G. H. 1995, ApJ, 441, 96

Moles, M., Márquez, I., \& Pérez, E. 1995, ApJ, 438, 604

Mulchaey, J., \& Regan, M. 1997, ApJ, 482, L135

Mulchaey, J., Regan, M., \& Kundu, A. 1997, ApJS, 110, 299

Peletier, R. F., Davies, R. L., Illingworth, G., Davis, L., \& Cawson, M. 1990, AJ, 100, 1091

\section{REFERENCES}

Peletier, R. F., Knapen, J. H., Shlosman, I., Pérez-Ramirez, D., Nadeau, D., Doyon, R., Rodriguez-Espinosa, J. M., \& Pérez-García, A. M. 1999, ApJS, in press (Paper I)

Phinney, E. S. P. 1994, in Mass-Transfer Induced Activity in Galaxies, ed. I. Shlosman (New York: Cambridge Univ. Press), 1

Regan, M. W., \& Mulchaey, J. S. 1999, AJ, 117, 2676

Sandage, A., \& Tammann, G. A. 1981, A Revised Shapley-Ames Catalog of Bright Galaxies (Publ. 635; 1st ed.; Washington: Carnegie Inst. of Washington)

Sellwood, J. A., \& Wilkinson, A. 1993, Rep. Prog. Phys., 56, 173

Shaw, M. A., Combes, F., Axon, D. J., \& Wright, G. S. 1993, A\&A, 273, 31

Shlosman, I. 1992, in ASP Conf. Ser. 31, Relationships between Active Galactic Nuclei and Starburst Galaxies, ed. A. V. Filippenko (San Francisco: ASP), 335

1996, in Proc. Nobel Symp. on Barred Galaxies and Circumnuclear Activity, ed. A. Sandqvist \& P. O. Lindblad (New York: Springer), 141

Shlosman, I., Begelman, M. C., \& Frank, J. 1990, Nature, 345, 679

Shlosman, I., Frank, J., \& Begelman, M. C. 1989, Nature, 338, 45

Shlosman, I., Peletier, R. F., \& Knapen, J. H. 1999, in preparation (Paper III)

Simkin, S. M. Su, H. J. \& Schwarz, M. P. 1980, ApJ, 237, 404

Spillar, E. J., Oh, S. P., Johnson, P. E., \& Wenz, M. 1992, AJ, 103, 793

Thronson, H., Jr., Hereld, M., Majewski, S., Greenhouse, M., Johnson, P., Spillar, E., Woodward, C. E., Harper, D. A., \& Rauscher, B. J. 1989, ApJ, 343,158 